\title{
Mechanism of circadian regulation of the NRF2/ARE pathway in renal ischemia-reperfusion
}

\author{
QIAN SUN ${ }^{1}, \mathrm{CHENG} \mathrm{ZENG}^{1}$, LI DU ${ }^{1}$ and $\mathrm{CHONG} \mathrm{DONG}^{2,3}$ \\ ${ }^{1}$ Department of Anesthesiology, Renmin Hospital of Wuhan University, Wuhan, Hubei 430060; ${ }^{2}$ Organ Transplantation Center, \\ Tianjin First Central Hospital; ${ }^{3}$ Tianjin Key Laboratory for Organ Transplantation, Tianjin 300192, P.R. China
}

Received June 29, 2020; Accepted November 24, 2020

DOI: $10.3892 /$ etm.2021.9622

\begin{abstract}
The nuclear erythroid 2-related factor 2(NRF2)/antioxidant response element (ARE) pathway has been shown to provide strong protection against oxidative stress injury induced by renal ischemia-reperfusion (IR). However, the endogenous regulatory mechanism of the NRF2/ARE pathway in renal IR injury is incompletely understood. A rat model of renal IR was established by occlusion of the bilateral renal pedicle for $45 \mathrm{~min}$, followed by reperfusion for $24 \mathrm{~h}$. Renal injury was assessed by light microscopy and levels of serum creatinine, blood urea nitrogen and neutrophil gelatinase-associated lipocalin was measured using enzyme-linked immunosorbent assay. Renal oxidative stress was also evaluated by measuring superoxide dismutase and malondialdehyde in renal tissues. Protein expression levels of brain and muscle ARNT-like 1 (BMAL1), nuclear factor erythroid 2-related factor 2 (NRF2), NAD(P)H dehydrogenase [quinone] 1 (NQO1), glutamate-cysteine ligase modifier (GCLM) and heme oxygenase 1 (HO1) in the kidney were determined by western blotting and immunohistochemistry. Reverse transcription-quantitative PCR was used to evaluate rhythmic transcription of the core clock genes (CLOCK and BMAL1) and the NRF2 gene. The nature of the binding of BMAL1 to the promoter regions in the NRF2 gene was assessed by chromatin immunoprecipitation assays in rat kidneys. BMAL1 was found to bind to the promoter of the NRF2 gene through an E-BOX element associated with strongly rhythmic activation of NRF2 in both the normal kidney and those exposed to IR. The ARE-regulated anti-oxidative stress protein was affected by the circadian rhythm of the NRF2 gene. As the NRF2 level was at a circadian nadir, the expression of the proteins NQO1, GCLM and HO1 was weakened, resulting in more serious renal oxidative stress injury and pathological and
\end{abstract}

Correspondence to: Professor Chong Dong, Organ Transplantation Center, Tianjin First Central Hospital, 24 Fukang Road, Nankai, Tianjin 300192, P.R. China

E-mail: dongchong@medmail.com.cn

Key words: circadian clock, nuclear erythroid 2-related factor 2, ischemia-reperfusion, renal injury functional impairment induced by IR. It can be concluded that the circadian rhythm of the NRF2/ARE pathway controlled by the circadian clock is essential for regulating antioxidant stress in renal IR injury, which might prompt new therapeutic strategies associated with the diurnal variability of human kidney disease, including renal transplantation.

\section{Introduction}

Circadian rhythm in mammals is associated with the periodic oscillation of clock genes. The principal pacemaker is $\mathrm{SCN}$, which can express circadian clock genes autonomously. Brain and muscle ARNT-like 1 (BMAL1) is the core promoter of circadian rhythm, which binds to circadian locomotor output cycles protein kaput (CLOCK) to form a BMAL1/CLOCK complex, which then initiates the transcription of the PER and CRY genes. A negative feedback loop is then activated by the increased numbers of PER/CRY complexes which inhibits the activity of BMAL1/CLOCK complexes (1). An increasing body of evidence has demonstrated that circadian clock genes regulate the anti-oxidative stress mechanisms, especially the NRF2/ARE pathway $(2,3)$.

NRF2 is recognized as the core transcription factor of anti-oxidative stress, able to activate a large number of protective proteins, in which ARE-regulated antioxidant proteins are most important, including $\mathrm{NAD}(\mathrm{P}) \mathrm{H}$ dehydrogenase [quinone] 1 (NQO1), glutamate-cysteine ligase modifier (GCLM) and heme oxygenase 1 (HO1) (4). BMAL1 can regulate the expression of NRF2 and its downstream antioxidant stress protein by binding to the PPAR promoter through an E-BOX element, aggregating the NRF2 protein together in a circadian rhythm, which involves the transcription of ARE and other key antioxidant proteins in a circadian rhythm (5). The cyclic activation of NRF2 plays a vital role through its rhythmic recruitment of the promoter of the targeted antioxidant gene, coordinating its ability to resist oxidative stress in renal disease $(6,7)$.

A previous study had confirmed that NRF2 plays an important role in ischemia-reperfusion (IR) injury as a key endogenous protective mechanism of oxidative stress (8). In addition, recent studies have found that clock genes can act as endogenous molecular regulators of the NRF2 redox pathway, participating in the pathological mechanism of pulmonary fibrosis, and affecting anti-oxidative response capability (9). 
However, the internal mechanisms of the circadian clock genes that regulate the NRF2-associated endogenous redox pathway or dysrhythmia of the NRF2/ARE pathway that affect the circadian clock in renal IR injury have not been defined. Thus, in the present study, the effect and endogenous regulatory mechanism of dysrhythmia via the NRF2/ARE was explored in the kidney for protection from oxidative stress induced by IR.

\section{Materials and methods}

Materials. In total, 50 male adult SD rats (220-250 g; 6-8 weeks old) were purchased from The Animal Center of Renmin Hospital of Wuhan University (Wuhan, China). Rats were housed in specific-pathogen-free (SPF) conditions at $22-24^{\circ} \mathrm{C}$, a relative humidity of $50 \pm 15 \%$, receiving standard laboratory chow and water. A total of $\geq 10$ days prior to experimentation, the rats were housed in a strict 12-h light/dark cycle [lights on at zeitgeber time (ZT) 0]. The experimental protocol of the present study was approved by the Ethics Committee of Renmin Hospital of Wuhan University and in accordance with the principles of Laboratory Animal Care by the National Institutes of Health (permit no. 8023).

Antibodies for BMAL1 and NRF2 were purchased from Abcam. Antibodies for NQO1, GCLM and HO1 were obtained from Sigma-Aldrich. $\beta$-actin and LaminB were purchased from Cell Signaling Technology, Inc. (cat. nos. 4970 and 13435, respectively), and horseradish peroxidase (HRP)-conjugated secondary antibodies were purchased from Santa Cruz Biotechnology, Inc. Blood urea nitrogen (BUN) and serum creatinine ( $\mathrm{Scr}$ ) were measured using an Olympus automatic analyzer and neutrophil gelatinase associated lipocalin (NGAL) levels were quantified using the corresponding enzyme-linked immunosorbent assay (ELISA) kit purchased from Elabscience, Inc. Superoxide dismutase (SOD) and malondialdehyde (MDA) assay kits were purchased from Nanjing Jiancheng Biochemicals Ltd.

Renal ischemia-reperfusion model. All rats were anesthetized by intraperitoneal injection of $2 \%$ pentobarbital sodium $(40 \mathrm{mg} / \mathrm{kg})$. Rats were immobilized and subsequently connected to an ECG monitor, after whom the trachea was cut and the animals mechanically ventilated. The IR model was established by bilateral renal pedicle occlusion for ischemia (45 min), followed by removal of the microvascular clip for $24 \mathrm{~h}$ reperfusion (10). Except for occlusion, the other surgical procedures in the $S$ Group were the same. The procedure was successful if the kidney turned from red to black after the pedicle occlusion, then black to red after gradual removal the clip. The surgery was considered successful if the rats regained consciousness after 1-3 h.

Histopathology of kidney tissue. The left kidney was sectioned, then fixed with $4 \%$ formaldehyde for $24 \mathrm{~h}$ at room temperature, then embedded in paraffin, from which $4-\mu \mathrm{m}$ sections were cut and stained with hematoxylin for $3 \mathrm{~min}$ and eosin for $60 \mathrm{sec}$ at room temperature. The slides were evaluated using light microscopy (original magnification, x200; Olympus BX50; Olympus Corporation). Renal histological assessment was conducted using a semi-quantitative scale, as described by Spandou et al (11): For each kidney, $\geq 100$ cortical tubules from 10 different regions were scored. Higher scores represented more severe damage, maximum score per tubule was 10 , scoring as follows: $0=$ Normal kidney; $1=$ minimal damage $(<5 \%$ involvement of the cortex or outer medulla); $2=$ mild damage $(5-25 \%$ involvement of the cortex or outer medulla); $3=$ moderate damage $(25-75 \%$ involvement of the cortex or outer medulla); $4=$ severe damage ( $>75 \%$ involvement of the cortex or outer medulla) (12).

Immunohistochemical assessment of NRF2 in the kidney. The streptavidin-biotin complex immunohistochemical technique has been described previously (13). It was used to detect NRF2 protein in paraffin-embedded kidney tissue sections by permeabilizing with $0.3 \%$ Triton X-100 (cat. no. P0096; Beyotime Institute of Biotechnology) at room temperature for 10 min, then blocked with $10 \%$ goat serum (cat. no. C0265; Beyotime Institute of Biotechnology) at $37^{\circ} \mathrm{C}$ for $10 \mathrm{~min}$, incubated overnight at $4^{\circ} \mathrm{C}$ with 1:400 NRF2 antibody (cat. no. ab92946; Abcam), incubated $30 \mathrm{~min}$ at $37^{\circ} \mathrm{C}$ with 1:500 Biotin-labeled secondary antibody (cat. no. A0277; Beyotime Institute of Biotechnology), incubated $1 \mathrm{~h}$ at room temperature with the 1:400 Streptavidin-HRP (cat. no. A0303; Beyotime Institute of Biotechnology) and dyed 2-5 min at room temperature with $\mathrm{DAB}+30 \% \mathrm{H}_{2} \mathrm{O}_{2}$. Positive expression in the cytoplasm and/or nucleus was stained brown (original magnification, x200; Olympus BX50; Olympus Corporation). The optical density of positive staining was semi-quantitatively evaluated using Image Pro ${ }^{\circledR}$ plus version 6.0 software (Media Cybernetics, Inc.).

Measurement of Scr, BUN and NGAL levels. After the end of IR, the right internal carotid artery of the rats was isolated, $2 \mathrm{ml}$ blood was collected from each group. Blood samples were collected at the end of reperfusion, centrifuged at 3,000 $\mathrm{x}$ for $10 \mathrm{~min}$ at $4^{\circ} \mathrm{C}$ and then serum was separated and stored at $-20^{\circ} \mathrm{C}$. Scr and BUN were measured using an Olympus automatic analyzer (AU5400; Olympus Corporation), and NGAL levels were measured using ELISA assay kits (cat. no. E-EL-R0662c; Elabscience, Inc.) as described previously (13).

Measurement of SOD activity and MDA levels in renal tissues. Renal tissues were harvested and immediately homogenized on ice in 5 volumes of normal saline. The homogenates were centrifuged at $1,200 \mathrm{x}$ for $10 \mathrm{~min}$ at $4^{\circ} \mathrm{C}$. SOD activity (cat. no. A001-3-2) and MDA levels (cat. no. A003-1-2) were measured using a chemical assay kit (cat. nos. A001-3-2 and A003-1-2, respectively; Nanjing Jiancheng Biochemicals Ltd.) in accordance with the manufacturer's protocol.

Western blot analysis. Cytoplasmic and nuclear proteins of the renal tissues were extracted using nuclear and cytoplasmic protein extraction kit (cat. no. P0028; Beyotime Institute of Biotechnology) according to the manufacturer's instructions. After measurement of the protein concentration using the bicinchoninic acid method, an equal quantity of $50 \mu \mathrm{g}$ protein was separated by $12 \%$ SDS-PAGE. After electrophoresis, proteins were transferred onto polyvinylidene difluoride membranes. Each membrane was blocked with 5\% nonfat milk for $2 \mathrm{~h}$ at room temperature, then incubated overnight at $4{ }^{\circ} \mathrm{C}$ with an appropriate primary antibody: BMAL1 (cat. nos. ab231793; 
Abcam), NRF2 (cat. nos. ab92946; Abcam), NQO1, GCLM or HO1 (cat. nos. N5288, SAB2100907 and 374087, respectively; Merck KGaA), each at 1:800 dilution). After repeated washing with TBS-T (containing $0.05 \%$ Tween-20) the membranes were incubated with the HRP-conjugated secondary antibodies (1:2,000; cat. no. sc2357; Santa Cruz Biotechnology, Inc.) for $2 \mathrm{~h}$ at room temperature. The immunoreactive bands were visualized by enhanced chemiluminescence (cat. no. NEL103E001EA; PerkinElmer, Inc.) and captured on X-ray films. The optical density of the bands was measured with Glyko ${ }^{\circledR}$ BandScan V4.0 imaging analysis system (http://bandscan.software.informer.com/).

$R N A$ extraction and reverse transcription-quantitative $(R T-q)$ $P C R$. Total RNA was isolated from renal tissue using an RNAeasy $^{\mathrm{TM}}$ animal RNA isolation kit (Beyotime Institute of Biotechnology). cDNA was synthesized at $42^{\circ} \mathrm{C}$ for $60 \mathrm{~min}$ and $70^{\circ} \mathrm{C}$ for $15 \mathrm{~min}$ using a BeyoRT ${ }^{\mathrm{TM}}$ First Strand cDNA synthesis kit (cat. no. D7166; Beyotime Institute of Biotechnology) according to the manufacturer's protocols. Quantitative real-time PCR (protocol: $50^{\circ} \mathrm{C}$ for $2 \mathrm{~min}, 95^{\circ} \mathrm{C}$ for $10 \mathrm{~min}$; 40 cycles of $95^{\circ} \mathrm{C}$ for $30 \mathrm{sec}$ and $60^{\circ} \mathrm{C}$ for $30 \mathrm{sec}$ ) was conducted using SYBR Green Master mix (Vazyme Biotech, Co., Ltd.) and primers for CLOCK (forward, 5'-TCAAGGCCAGAGTTCATCGT-3' and reverse, 5'-GAG TTGGGCTGTGATCGAAC-3'), BMAL1 (forward, 5'-TGA ACCAGACAATGAGGGCT-3' and reverse, 5'-TATGCC AAAATAGCCGTCGC-3'), and NRF2 (forward, 5'-CCC ATTGAGGGCTGTGAT-3' and reverse, 5'-TTGGCT GTGCTTTAGGTC-3'). Results were quantified using the $2^{-\Delta \Delta \mathrm{Cq}}$ method (14) and normalized against $\beta$-actin (forward primer, 5'-CACGATGGAGGGGCCGGACTCATC-3'; reverse, 5'-TAAAGACCTCTATGCCAACACAGT-3').

Chromatin immunoprecipitation (ChIP) assays. Following homogenization, the kidney tissue was cross-linked with $1 \%$ (v/v) formaldehyde for $10 \mathrm{~min}$ at $37^{\circ} \mathrm{C}$ and cell nuclei lyzed using Bioruptor ultrasound (500 bp; $25 \%$ power; $4.5 \mathrm{sec}$ impact, $9 \mathrm{sec}$ interval, 14 times in total) to obtain chromatin. The chromatin was incubated with the following antibodies at $4{ }^{\circ} \mathrm{C}$ overnight as follows: $1 \mu \mathrm{g} / \mathrm{ml}$ anti-NRF2 (cat. no. ab137550; Abcam), $4 \mu \mathrm{g} / \mathrm{ml}$ anti-BMAL1 (cat. no. ab231793; Abcam), and $1 \mu \mathrm{g} / \mathrm{ml}$ control IgG (cat. no. ab172730; Abcam). Target DNA fragment were obtained by protein $G$ magnetic Dynabead (cat. no. 10003D; Life Technologies) immunoprecipitation, then reverse chromatin cross-linking with $5 \mathrm{M} \mathrm{NaCl}$ overnight at $65^{\circ} \mathrm{C}$ and digestion by protease $\mathrm{K}$ (cat. no. 1.24568; Sigma-Aldrich; Merck KGaA), after which the DNA was eluted. Using RT-qPCR technology, the E-BOX (forward 5'-GAGCCCAGGGCACGTGGGAGA AGTGG-3' and reverse, 5'-CCACTTCTCCCACGTGCCCTGGGCTC-3') of the promoter region was amplified and quantified.

Statistical analysis. All outcome measurements were expressed as means \pm SD values $(n=5)$ and analyzed using Graph Pad Prism 6.0 (GraphPad Software, Inc.). Statistical significance of differences among groups was determined by a one-way ANOVA with Tukey's post hoc test. Comparison of two groups was evaluated using a t-test. $\mathrm{P}<0.05$ was considered to indicate a statistically significant difference.

\section{Results}

Circadian rhythm of NRF2 gene in the kidney. In order to explore the circadian rhythm of NRF2 protein expression in normal kidneys, total NRF2 protein expression levels were evaluated in the normal rat kidneys that were collected every $4 \mathrm{~h}$ after ZT0. Western blot analysis indicated that total NRF2 protein expression levels in the $\mathrm{S}$ group displayed a strong circadian rhythm (Fig. 1A). The peak of NRF2 protein expression was between ZT0 and ZT4, with a trough between ZT12 and ZT16 (Fig. 1A). NRF2 protein translocation into the nucleus initiates activation of downstream antioxidant proteins. It was found that the nuclear NRF2 protein expression in normal kidney tissue also exhibited a circadian rhythm, with an amplitude and peak phase that mirrored total NRF2 protein expression (Fig. 1B). In addition, immunostaining of NRF2 protein indicated that the nuclear NRF2 protein expression in renal tubular epithelial cells displayed clear diurnal variability (Fig. 1C). Compared with ZT0, the expression level of nuclear NRF2 protein in the ZT12 group was weaker $(\mathrm{P}<0.05$; Fig. 1C). In order to further explore the circadian rhythm of the NRF2 gene transcription, quantitative PCR was used to measure mRNA of the core clock genes CLOCK and BMAL1 in the rat kidney, finding that they displayed a strong endogenous circadian rhythm, corresponding to that of NRF2 mRNA (Fig. 1D). Together, these results strongly suggest that there is a significant circadian rhythm in the NRF2 gene in the kidney that is closely associated with the circadian clock of the body.

Diurnal variability of oxidative stress in the kidney following IR injury. The present study investigated whether the rhythmic expression of the oxidative stress and core protein NRF2 induced diurnal variability in the anti-oxidant stress capability in renal IR injury. It was hypothesized that the rhythmic expression of NRF2 may regulate the downstream ARE-regulated genes affecting IR injury, which has diurnal variability. In order to verify this, the rat renal IR model was established at a time point close to the peak or trough of NRF2 protein expression. The peak of NRF2 protein expression was between ZT0 and ZT4, and a trough between ZT12 and ZT16. In order to fit into a 12-h light/12-h dark cycle, ZT0 and ZT12 were selected as the two time points for sampling. As shown in Fig. 2A, following 45 min of ischemia, reperfusion significantly decreased SOD activity and increased MDA levels of renal tissues in the ZT12 group compared with those in the ZT0 Group $(\mathrm{P}<0.05)$. The protein expression levels of NQO1, GCLM and HO1 exhibited clear diurnal variability, and the expression in the ZT0 group was significantly higher compared with that of the ZT12 group $(\mathrm{P}<0.05$ for all three genes; Fig. 2B).

Diurnal variability of renal injury induced by IR. As expected, and displayed in Fig. 3A, following 24-h reperfusion, characteristic histological changes to renal tubules, including tubular epithelial edema and swelling, lumen dilation, epithelial simplification, nuclear necrosis, cytoplasmic translucency, and vacuolation were observed in the IR groups. Histological changes in ZT12 were significantly increased compared with the ZT0 IR group. Compared with the normal kidney tissue, renal histology scores in the IR group were 
A
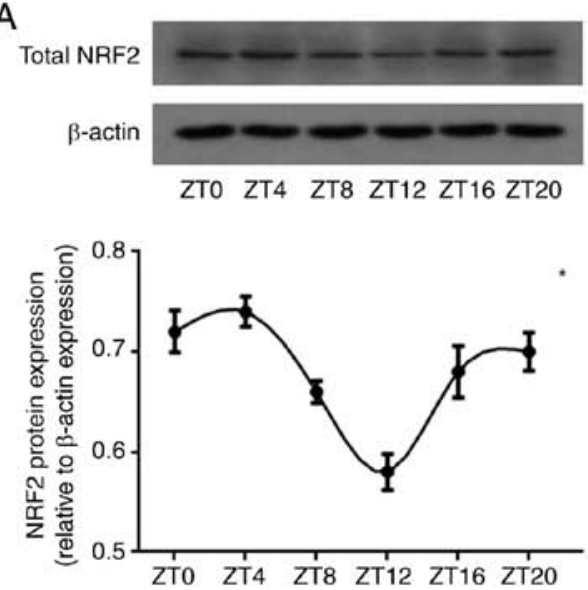

B
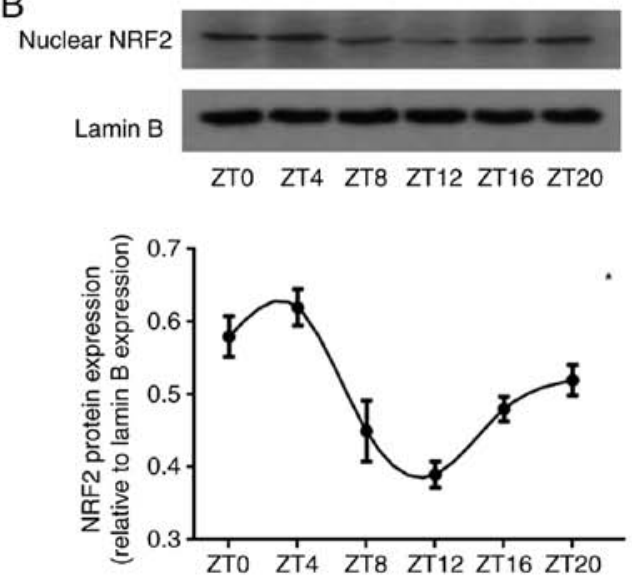

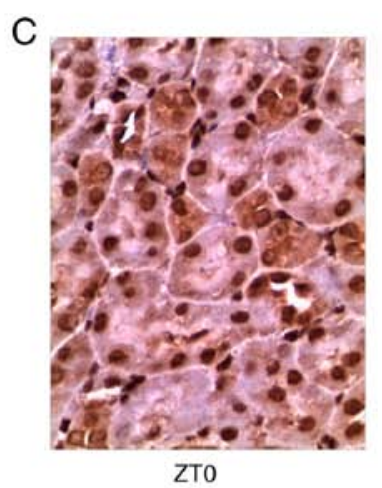

ZTO

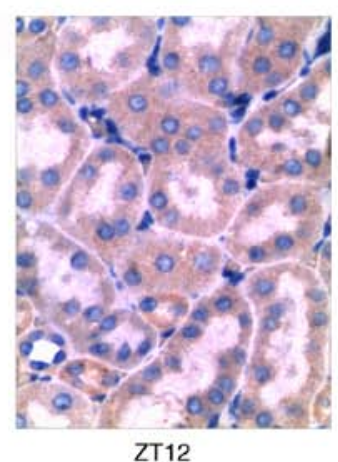

$\mathrm{ZT} 12$
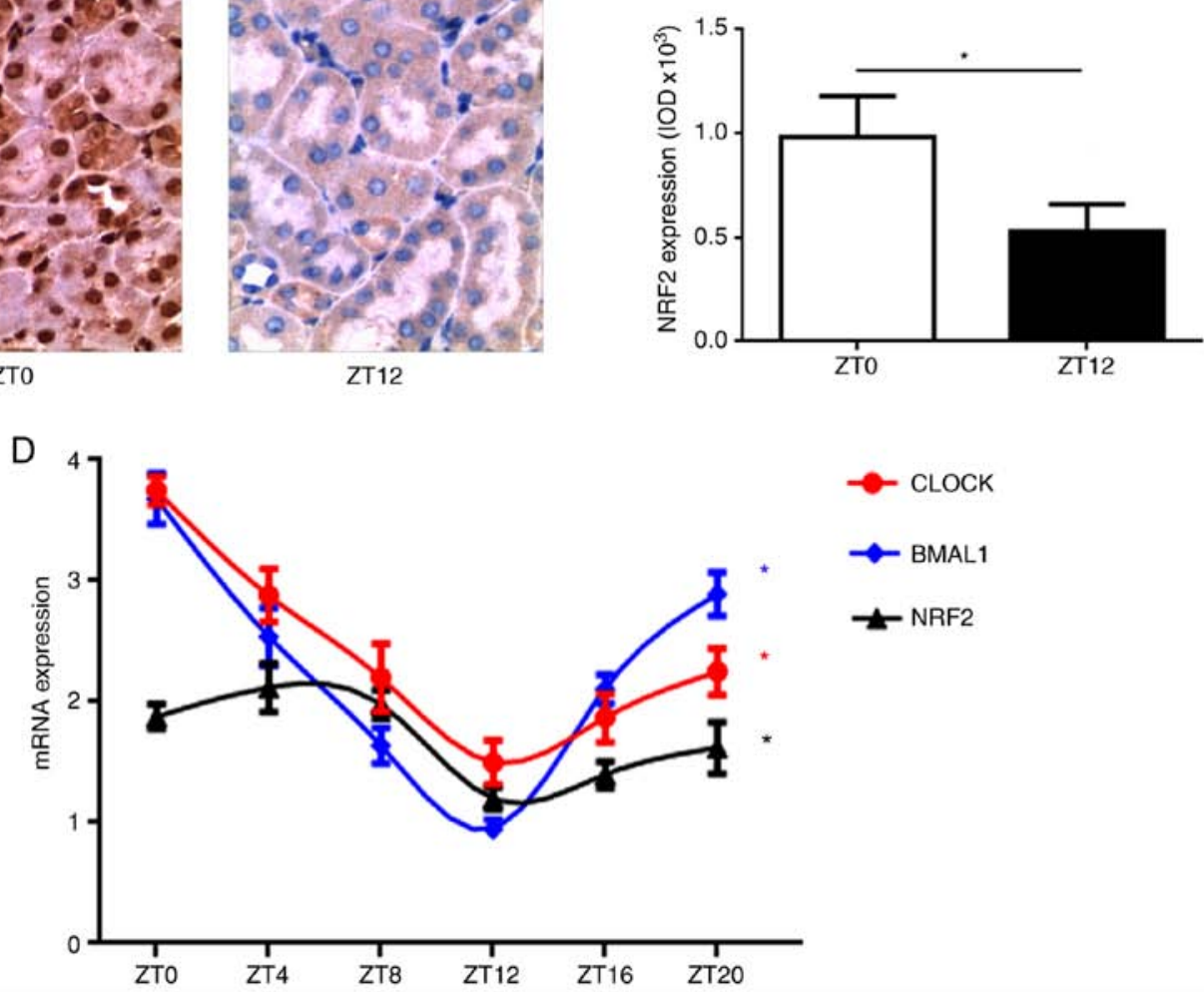

Figure 1. Circadian rhythm of NRF2 gene in the kidney. (A) Total NRF2 protein expression levels by western blot analysis. A strong circadian rhythm of total NRF2 protein expression was revealed in normal kidney. The peak of NRF2 protein expression was between ZT0 and ZT4, with a trough between ZT12 and ZT16. (B) Nuclear NRF2 protein expression levels by western blot analysis. The nuclear NRF2 protein expression in normal kidney tissue also exhibited a circadian rhythm, with an amplitude and peak phase that mirrored total NRF2 protein expression. NRF2 densitometry (mean \pm SD; $n=5)$ was normalized to $\beta$-actin or lamin B. One-way ANOVA for the effect of time, ${ }^{*} \mathrm{P}<0.05$. (C) NRF2 protein expression levels by immunostaining. Positive expression in the cytoplasm and/or nucleus was stained brown (original magnification, $\mathrm{x} 200$ ). The nuclear NRF2 protein expression in renal tubular epithelial cells displayed clear diurnal variability. Compared with ZT0, the expression of nuclear NRF2 protein at ZT12 group was weaker. Data presented as mean \pm SD, $n=5$. ${ }^{*} \mathrm{P}<0.05$. (D) The CLOCK, BMAL1 and NRF2 mRNA expression by quantitative PCR. CLOCK and BMAL1 mRNA expression displayed a strong endogenous circadian rhythm, corresponding to that of NRF2 mRNA in normal kidney. Data (mean $\pm \mathrm{SD} ; \mathrm{n}=5$ ) were normalized to GAPDH. One-way ANOVA for the effect of time, ${ }^{*} \mathrm{P}<0.05$. NRF2, nuclear factor erythroid 2-related factor 2.

significantly higher $(\mathrm{P}<0.05)$. Compared with ZT0, the score was significantly higher when the IR model was established at ZT12 $(\mathrm{P}<0.05)$. Furthermore, Scr, BUN and NAGL levels were higher in the ZT12 IR group compared with the ZT0 IR group, indicating a higher induction of acute kidney injury (Fig. 3B). Overall, the results confirm the hypothesis that rhythmic activation of ARE-regulated antioxidant protein induces diurnal variability of the anti-oxidant stress capability in renal IR injury.

Binding of BMAL1 to the NRF2 gene through an E-BOX region in the kidney following $I R$ injury. The rhythmic recruitment and activation of NRF2 and downstream antioxidant proteins led to the probing of whether and how the circadian 

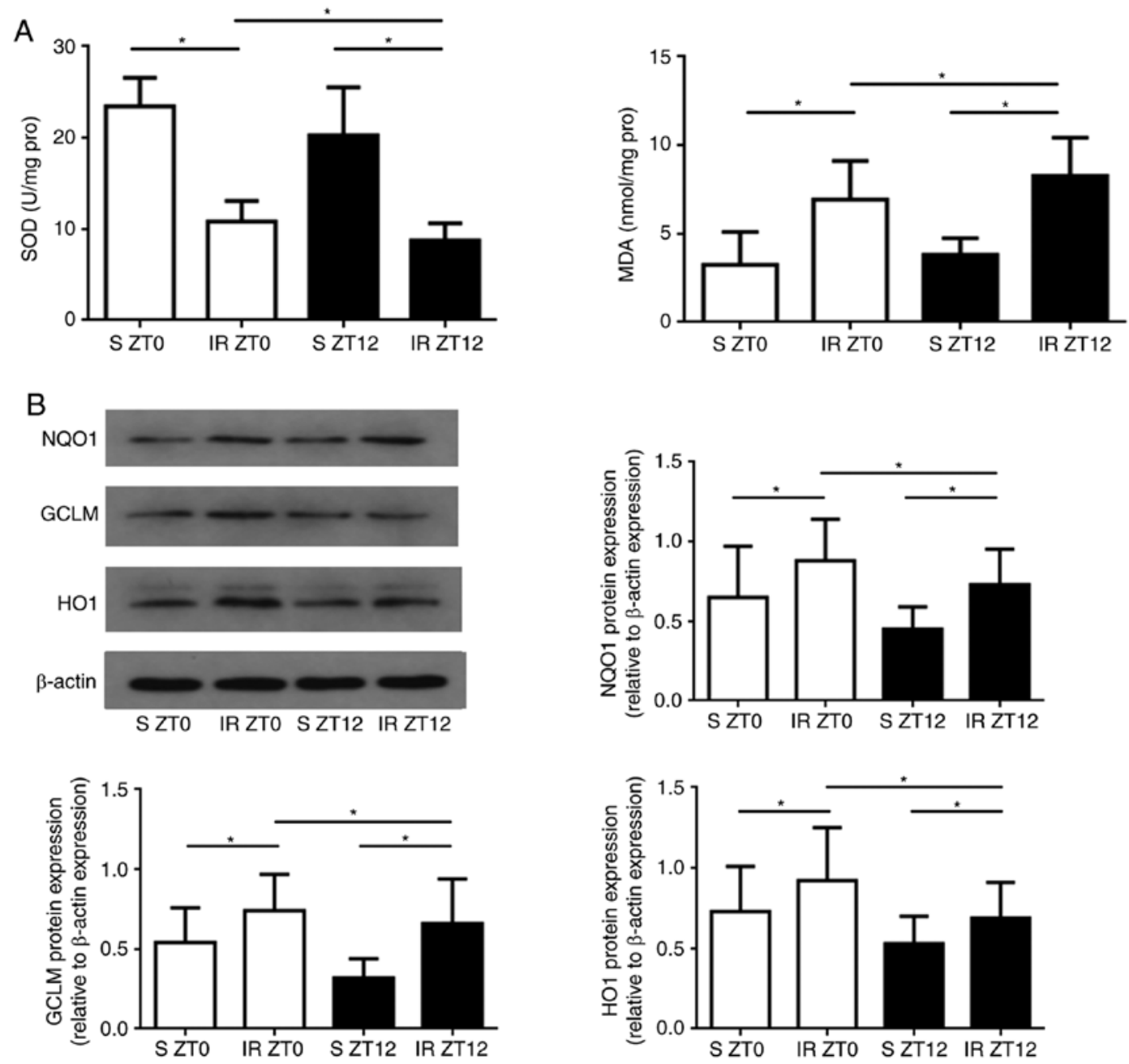

Figure 2. Diurnal variability of oxidative stress in the kidney following IR injury. (A) The SOD activity and MDA levels in renal tissues. SOD (U/mg pro) activity decreased and MDA (nmol/g pro) levels increased in the ZT12 group compared with those in ZT0 group (data presented as mean \pm SD; $n=5$; ${ }^{*} \mathrm{P}<0.05$ ). (B) The NQO1, GCLM and HO1 protein expression levels by western blot analysis. The expression in ZT0 group was significantly higher compared with that of ZT12 group (data presented as mean $\pm \mathrm{SD} ; \mathrm{n}=5 ;{ }^{*} \mathrm{P}<0.05$ for all three proteins). IR, ischemia-reperfusion; SOD, superoxide dismutase; MDA, malondialdehyde; $\mathrm{NQO1}$, NAD(P)H dehydrogenase [quinone] 1; GCLM, glutamate-cysteine ligase modifier; HO1, heme oxygenase 1.

clock regulated the NRF2/ARE pathway in renal IR injury. To explore the role of BMAL1 rhythms in coupling the rhythmic activation of the NRF2 gene to diurnal variability of IR injury in the kidney, the expression of BMAL1 and nuclear NRF2 protein were measured. After reperfusion, BMAL1 and nuclear NRF2 protein expression were both significantly higher when IR was established at ZT0 compared with ZT12 $(\mathrm{P}<0.05$; Fig. 4A and $\mathrm{B})$.

In order to further verify the hypothesis that the core clock gene may bind rhythmically to the promoter of the NRF2 gene, we conducted temporal ChIP assays in the rat kidney tissue. The results demonstrated that the BMAL1 gene bound to the promoter of the NRF2 gene through the E-BOX region. It was observed that the binding of BMAL1 to the NRF2 gene at ZT0 was significantly higher compared with that of ZT12 in both S and IR groups ( $\mathrm{P}<0.05$; Fig. 4C), corresponding to the diurnal variability of the NRF2 expression. Together, the results indicate that the core clock gene BMAL1 controls the rhythmic expression of the NRF2 gene directly through the E-BOX region of the kidney following IR injury.

\section{Discussion}

In the present study, it was found that the circadian rhythm of the NRF2/ARE pathway controlled by the circadian clock is essential for the regulation of antioxidant stress in renal IR injury. Strong rhythmic binding of BMAL1 specifically to an E-BOX region was detected in the gene promoters of NRF2. Subsequently, the rhythmic recruitment and activation of NRF2 protein played a critical role in the rhythmic expression of downstream antioxidant proteins (such as NQO1, GLCM and HO1), which are involved in renal IR injury. The results identified a pivotal role for the circadian rhythm of the NRF2/ARE pathway controlled by the circadian clock, which is essential in protecting against oxidative stress injury in renal IR.

As a peripheral organ, the kidney has an independent biological clock system, that regulates its physiological function and exhibits diurnal variability, including its blood pressure, glomerular filtration rate, and urinary sodium excretion. Recent studies have confirmed that the circadian rhythm of kidney function and the phase of clock gene expression (such as those of CLOCK, BMAL1 and Per1/2) are 
A

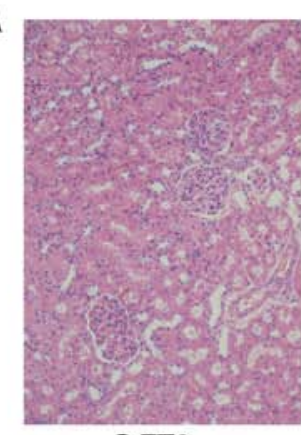

S ZTO

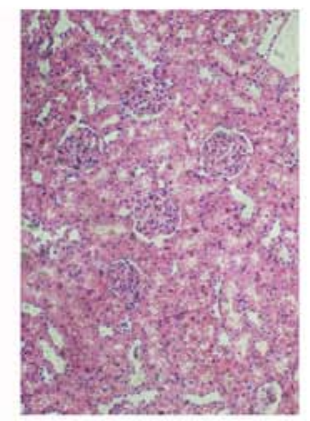

IR ZTO

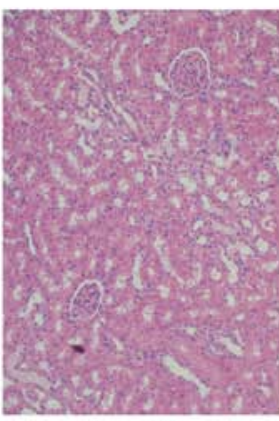

S ZT12

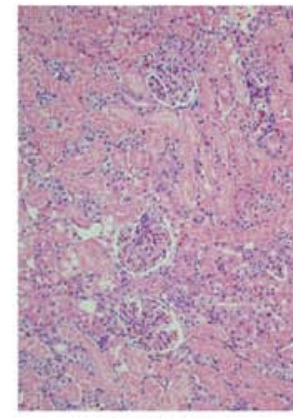

IR ZT12
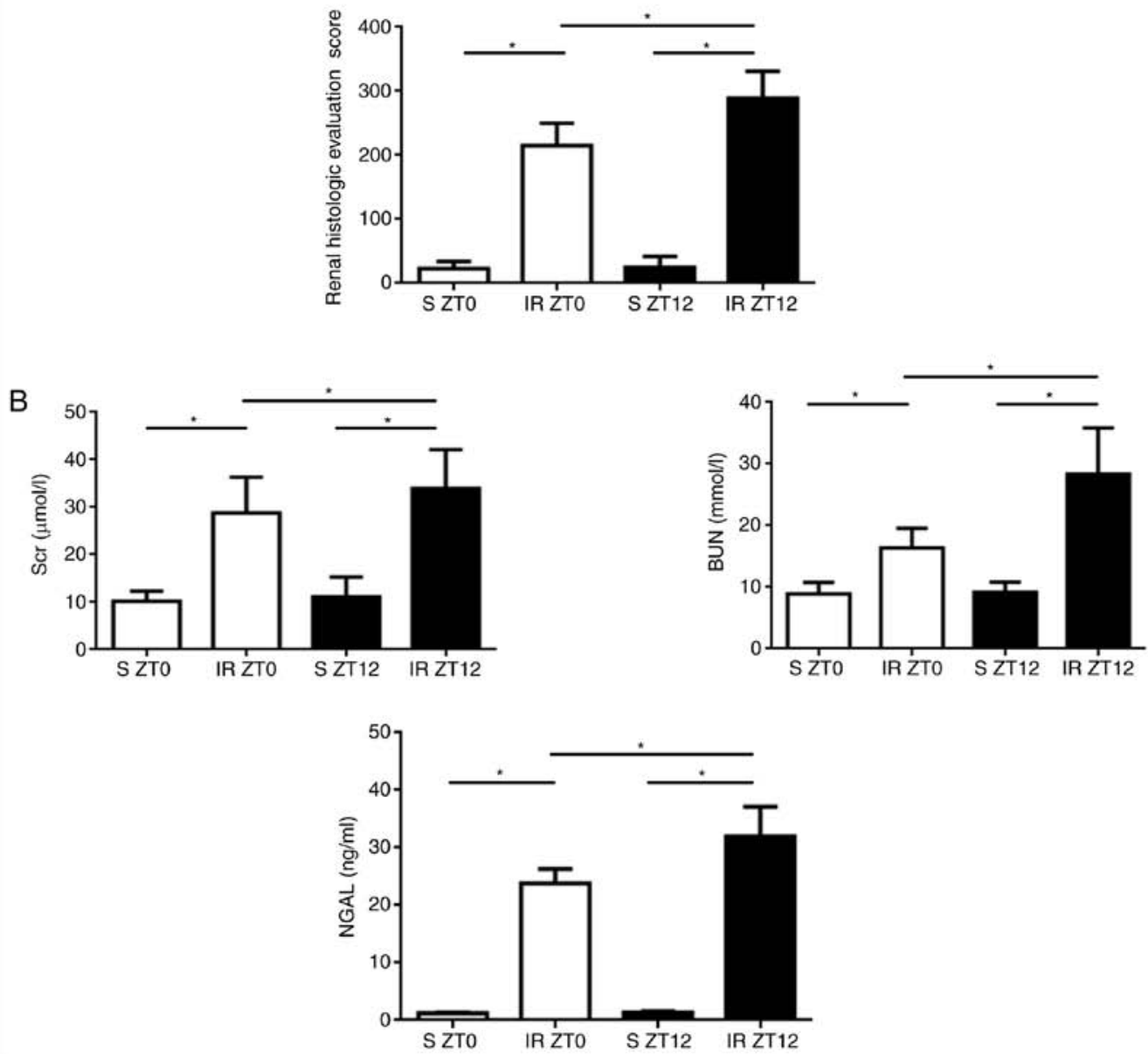

Figure 3. Diurnal variability of renal injury induced by IR. (A) Renal IR injury assessment (original magnification, x200). Following 24-h reperfusion, characteristic histological changes in renal tubule, including tubular epithelial edema and swelling, lumen dilation, epithelial simplification, nuclear necrosis, cytoplasmic translucency and vacuolation were observed in IR groups. Histological changes of ZT12 increased significantly compared with those of ZT0 in the IR groups. Renal histologic evaluation score was higher when the IR model was established at ZT12 (data presented as mean \pm SD; $n=5$; ${ }^{*} P<0.05$ ). (B) The Scr, BUN and NAGL levels determined by enzyme-linked immunosorbent assay. The levels of ZT0 group were significantly higher compared with those of the ZT12 group (data presented as mean $\pm \mathrm{SD} ; \mathrm{n}=5 ;{ }^{*} \mathrm{P}<0.05$ for all three indicators). IR, ischemia-reperfusion; Scr, serum creatinine; BUN, blood urea nitrogen; NAGL, neutrophil gelatinase associated lipocalin.

coordinated, a critical molecular mechanism that maintains the physiological function of the kidney (15). Furthermore, dysregulation of circadian gene expression can damage renal function and significantly influence systemic diseases, such as hypertension, sleep cycle disorder and cancer (16). As shown in Fig. 1, mRNA expression levels of core clock genes, including CLOCK and BMAL1, exhibited robust endogenous circadian rhythm in the normal kidney.

A report in the Lancet demonstrated that patients undergoing cardiac surgery during the daytime exhibit a different tolerance to IR injury and degree of myocardial injury compared with those during the night, due to the rhythmic expression of clock genes (17). A similar observation has been reported in animals in models of IR (18-20), suggesting that the rhythmic expression of the clock gene plays an important role in oxidative stress injury following IR, although the underlying mechanism is unclear. In the present study, it was found that the rhythm of clock genes and indicators of oxidative stress within the kidney were disordered following IR, which was accompanied by the pathological and functional 
A
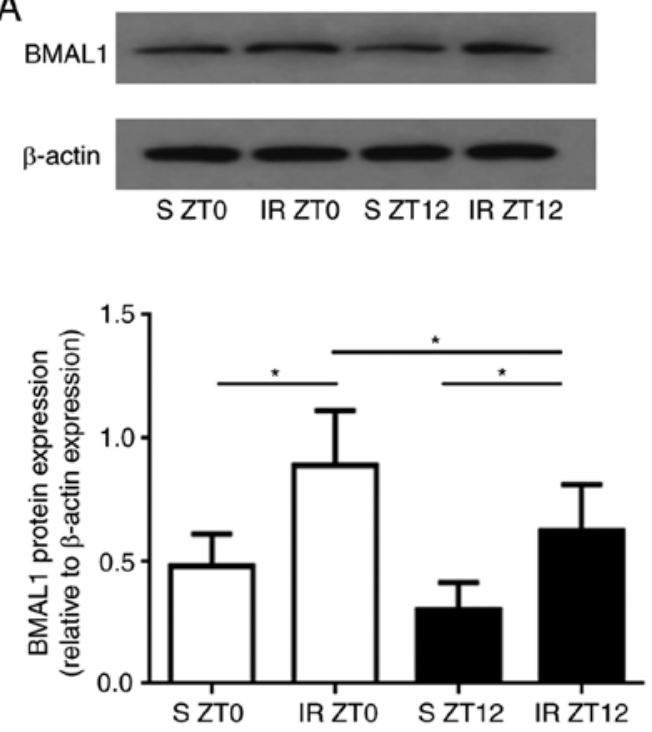

B

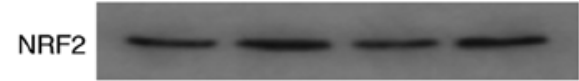

Lamin B

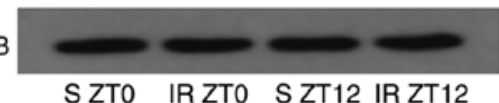

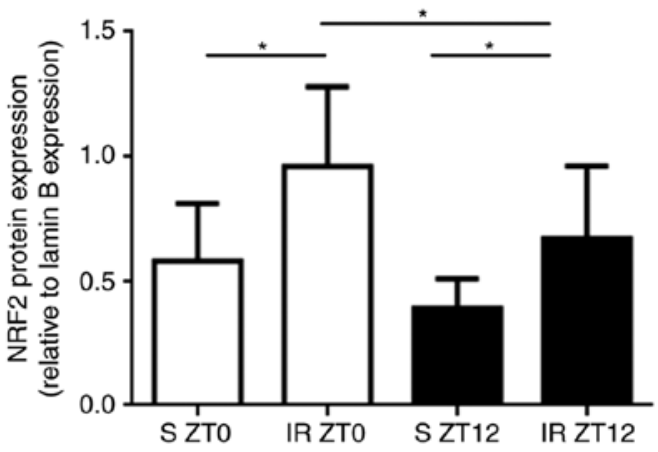

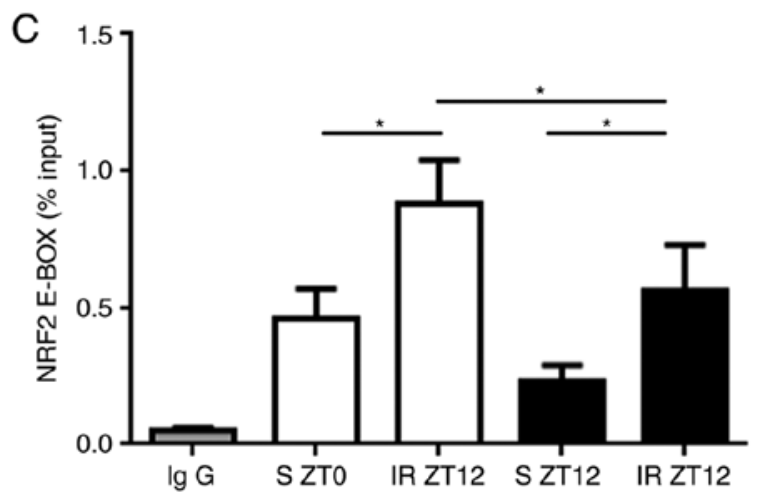

Figure 4. Binding of BMAL1 to the NRF2 gene through an E-BOX region in the kidney following IR injury. (A and B) The BMAL1 and nuclear NRF2 protein expression levels in renal IR injury by western blot analysis. After reperfusion, BMAL1 and nuclear NRF2 protein expression were both significantly higher when IR was established at ZT0 compared with ZT12. Data presented as mean $\pm \mathrm{SD}, \mathrm{n}=5$. ${ }^{*} \mathrm{P}<0.05$. (C) The E-BOX expression levels by temporal chromatin immunoprecipitation assays. The binding of BMAL1 to the NRF2 gene at ZT0 was significantly higher compared with that of ZT12 in both $\mathrm{S}$ and IR groups. Data presented as mean $\pm \mathrm{SD} ; \mathrm{n}=5$; "P<0.05. NRF2, nuclear factor erythroid 2-related factor 2; BMAL1, brain and muscle ARNT-like 1; IR, ischemia-reperfusion.

impairment of the kidney. Therefore, it was speculated that IR may lead to the disorder of the circadian rhythm of the renal clock gene, impairing the consistency between clock genes, which may represent the endogenous molecular mechanism causing renal oxidative stress injury. IR injury is an inevitable pathological process in renal transplantation. Clinical observation has shown that melatonin secretion, blood pressure, and fluctuation in body temperature, time of sleep onset, total duration of sleep time and its depth did not improve in renal transplant patients, and the rhythmic variations in urinary hormone excretion were also disturbed $(15,21)$. This may also be closely associated with the incidence of cardiovascular disease, deterioration in renal allograft function and long-term survival in patients undergoing renal transplant.

In previous research, it was confirmed that the NRF2/ARE pathway plays a vital role in defense against oxidation that balances oxidative stress induced by ROS in renal IR injury (13,22). Cyclic activation and rhythmic recruitment of NRF2 protein are controlled by the core clock genes CLOCK and BMAL1. The rhythmic activation of the Nrf2/ARE pathway may be a key process for the downstream expression of antioxidant proteins for effective removal of ROS and inhibition of tissue damage (23). The results of the present study indicate that activation of NRF2 results in a change in the amplitude of circadian rhythm and periodicity, with associated variation in the rhythmic expression of CLOCK and BMAL1 in both normal and IR kidney tissue. The transactivation of NRF2 is regulated by the binding of BMAL1 to the NRF2 promoter in the E-BOX region, contributing to the rhythmic activation of the NRF2/ARE pathway and rhythmic expression of downstream antioxidant proteins (24). Thus ChIP assay was used to identify that, under physiological conditions, the recruitment and activation of NRF2 protein is especially controlled by the core clock protein BMAL1 in the kidney through its binding to NRF2. The data highlight further evidence that the dysrhythmia of NRF2 in the kidney has wide implications for renal pathological and functional impairment induced by IR and together with ARE redox-mediated regulation of antioxidant proteins, by detecting which can indirectly reflect the activation of NRF2/ARE pathway, including of 
NQO1, GCLM and HO1. From these observations, it appears that NRF2 may be a vital mechanism between the disorder of clock gene rhythm and diurnal oscillation of redox balance in renal IR injury. In the circadian pathway, IR-induced upregulation of NRF2 via BMAL1/CLOCK-mediated transactivation results in the transactivation of ARE-associated antioxidant proteins.

In conclusion, it was demonstrated that endogenous circadian clock genes regulating the NRF2/ARE pathway are associated with an anti-oxidative stress protection mechanism in the rat kidney following IR. The data indicate that the core clock gene BMAL1 plays a vital role in regulating the recruitment and activation of the NRF2 gene in the kidney. In addition, dysrhythmia of the NRF2/ARE pathway may affect the expression of downstream antioxidant proteins (such as NQO1, GLCM and HO1) conferring the rhythmic regulation to alter susceptibility to oxidative stress induced by IR in the kidney.

Limitation and future direction. The role of NRF2/ARE pathway in renal I/R injury has been confirmed by using NRF2 agonists and inhibitors in our previous study (25). The present study focused on the effect of the clock gene on NRF2 and its downstream substrates. Since the clock gene has no specific agonists or antagonists, future studies should employ specific gene knockout or gene mutations in mice in vivo, and gene silencing in primary cells in vitro, in order to establish that the inhibition of core clock genes such as CLOCK and BMAL1 affect the expression of NRF2, and so represent as potential therapeutic targets.

\section{Acknowledgements}

Not applicable.

\section{Funding}

This work was supported by the Independent Research Projects of Wuhan University (grant no. 2042018kf0100) and National Natural Science Foundation of China (grant no. 82072140).

\section{Availability of data and materials}

The datasets used and/or analyzed during the current study are available from the corresponding author on reasonable request.

\section{Authors' contributions}

QS and CD designed the study; CZ performed the majority of experiments; LD analyzed the data; QS wrote the manuscript; Illustrations and proofreading was performed by CD. QS and $\mathrm{CZ}$ can authenticate the raw data. All authors read and approved the final manuscript.

\section{Ethics approval and consent to participate}

The experimental protocol of the present study was approved by the Ethics Committee of Renmin Hospital of Wuhan University (Wuhan, China) and in accordance with the principles of Laboratory Animal Care by the National Institutes of Health (permit no. 8023).

\section{Patient consent for publication}

Not applicable.

\section{Competing interests}

The authors declare that they have no competing interests.

\section{References}

1. Bollinger T and Schibler U: Circadian rhythms-from genes to physiology and disease. Swiss Med Wkly 144: w13984, 2014.

2. Wilking M, Ndiaye M, Mukhtar H and Ahmad N: Circadian rhythm connections to oxidative stress: Implications for human health. Antioxid Redox Signal 19: 192-208, 2013.

3. Pei JF, Li XK, Li WQ, Gao Q, Zhang Y, Wang XM, Fu JQ, Cui SS, Qu JH, Zhao X, et al: Diurnal oscillations of endogenous $\mathrm{H}_{2} \mathrm{O}_{2}$ sustained by $\mathrm{p} 66^{\text {Shc }}$ regulate circadian clocks. Nat Cell Biol 21: 1553-1564, 2019.

4. Tonelli C, Chio IIC and Tuveson DA: Transcriptional regulation by Nrf2. Antioxid Redox Signal 29: 1727-1745, 2018.

5. Tamaru T, Hattori M, Ninomiya Y, Kawamura G, Varès G, Honda K, Mishra DP, Wang B, Benjamin I, Sassone-Corsi P, et al: ROS stress resets circadian clocks to coordinate pro-survival signals. PLoS One 8: e82006, 2013.

6. Desvergne A, Ugarte N, Radjei S, Gareil M, Petropoulos I and Friguet B: Circadian modulation of proteasome activity and accumulation of oxidized protein in human embryonic kidney HEK 293 cells and primary dermal fibroblasts. Free Radic Biol Med 94: 195-207, 2016.

7. Wible RS, Ramanathan C, Sutter CH, Olesen KM, Kensler TW, Liu AC and Sutter TR: NRF2 regulates core and stabilizing circadian clock loops, coupling redox and timekeeping in Mus musculus. Elife 7: e31656, 2018.

8. Sun Q, Shen ZY, Duan WN, Meng QT and Xia ZY: Mechanism of myocardial ischemia/reperfusion-induced acute kidney injury through DJ-1/Nrf2 pathway in diabetic rats. Exp Ther Med 14: 4201-4207, 2017.

9. Pekovic-Vaughan V, Gibbs J, Yoshitane H, Yang N, Pathiranage D, Guo B, Sagami A, Taguchi K, Bechtold D, Loudon A, et al: The circadian clock regulates rhythmic activation of the NRF2/glutathione-mediated antioxidant defense pathway to modulate pulmonary fibrosis. Genes Dev 28: 548-560, 2014.

10. Firsov D and Bonny O: Circadian rhythms and the kidney. Nat Rev Nephrol 14: 626-635, 2018.

11. Spandou E, Tsouchnikas I, Karkavelas G, Dounousi E, Simeonidou C, Guiba-Tziampiri O and Tsakiris D: Erythropoietin attenuates renal injury in experimental acute renal failure ischaemic/reperfusion model. Nephrol Dial Transplant 21: 330-336, 2006.

12. Chen R, Zeng Z, Zhang YY, Cao C, Liu HM, Li W, Wu Y, Xia ZY, Ma D and Meng QT: Ischemic postconditioning attenuates acute kidney injury following intestinal ischemia-reperfusion through Nrf2-regulated autophagy, anti-oxidation, and anti-inflammation in mice. FASEB J 34: 8887-8901, 2020.

13. Sun Q, Meng QT, Jiang Y, Liu HM, Lei SQ, Su WT, Duan WN, Wu Y, Xia ZY and Xia ZY: Protective effect of ginsenoside $\mathrm{Rb} 1$ against intestinal ischemia-reperfusion induced acute renal injury in mice. PLoS One 8: e80859, 2013.

14. Livak KJ and Schmittgen TD: Analysis of relative gene expression data using real-time quantitative PCR and the 2(-Delta Delta C(T)) method. Methods 25: 402-408, 2001.

15. Solocinski K and Gumz ML: The circadian clock in the regulation of renal rhythms. J Biol Rhythms 30: 470-486, 2015.

16. Johnston JG and Pollock DM: Circadian regulation of renal function. Free Radic Biol Med 119: 93-107, 2018.

17. Montaigne D, Marechal X, Modine T, Coisne A, Mouton S, Fayad G, Ninni S, Klein C, Ortmans S, Seunes C, et al: Daytime variation of perioperative myocardial injury in cardiac surgery and its prevention by Rev-Erb $\alpha$ antagonism: A single-centre propensity-matched cohort study and a randomized study. Lencet 391: 59-69, 2018. 
18. Durgan DJ, Pulinilkunnil T, Villegas-Montoya C, Garvey ME, Frangogiannis NG, Michael LH, Chow CW, Dyck JR and Young ME: Short communication: Ischemia/reperfusion tolerance is time-of-day-dependent: Mediation by the cardiomyocyte circadian clock. Circ Res 106: 546-550, 2010.

19. Rotter D, Grinsfelder DB, Parra V, Pedrozo Z, Singh S, Sachan N and Rothermel BA: Calcineurin and its regulator, RCAN1, confer time-of-day changes in susceptibility of the heart to ischemia/reperfusion. J Mol Cell Cardiol 74: 103-111, 2014.

20. Beker MC, Caglayan B, Yalcin E, Caglayan AB, Turkseven S, Gurel B, Kelestemur T, Sertel E, Sahin Z, Kutlu S, et al: Time-of-day dependent neuronal injury after ischemic stroke: Implication of circadian clock transcriptional factor Bmall and survival kinase AKT. Mol Neurobiol 55: 2565-2576, 2018.

21. Russcher M, Nagtegaal JE, Nurmohamed SA, Koch BC, van der Westerlaken MM, van Someren EJ, Bakker SJ, Ter Wee PM and Gaillard CA: The effects of kidney transplantation on sleep, melatonin, circadian rhythm and quality of life in kidney transplant recipients and living donors. Nephron 129: 6-15, 2015.
22. Sun Q, Meng QT, Jiang Y and Xia ZY: Ginsenoside Rb1 attenuates intestinal ischemia reperfusion induced renal injury by activating Nrf2/ARE pathway. Molecules 17: 7195-7205, 2012.

23. Yang G, Wright CJ, Hinson MD, Fernando AP, Sengupta S, Biswas C, La P and Dennery PA: Oxidative stress and inflammation modulate Rev-erba signaling in the neonatal lung and affect circadian rhythmicity. Antioxid Redox Signal 21: 17-32, 2014.

24. Wende AR, Young ME, Chatham J, Zhang J, Rajasekaran NS and Darley-Usmar VM: Redox biology and the interface between bioenergetics, autophagy and circadian control of metabolism. Free Radic Biol Med 100: 94-107, 2016.

25. Cheng Z, Qian S, Qingtao M, Zhongyuan X and Yeda X: Effects of ATRA on diabetic rats with renal ischemia-reperfusion injury. Acta Cir Bras 35: e202000106, 2020. 